\title{
Experimental Study on Improvement of Marine Soft Soil with Alkali Residue
}

\author{
SHAO Yong, ${ }^{*}$, LIU Xiao-li, ZHU Jin-jun \\ Lianyungang Technical College, Department of Architectural Engineering, 222006, Jiangsu, China
}

\begin{abstract}
Industrial alkali slag is the discharge waste in the process of alkali production. About one million tons of alkali slag is discharged in China in one year. It is a burden on the environment, whether it is directly stacked or discharged into the sea. If we can realize the use of resources, it is a multi-pronged move, so alkali slag is used to improve solidified marine soft soil in this paper. The test results show that the alkali residue can effectively improve the engineering properties of marine soft soil. Among them, the unconfined compressive strength and compressive modulus are increased by about 10 times, and the void ratio and plasticity index can all reach the level of general clay. It shows that alkali slag has the potential to improve marine soft soil and can be popularized in engineering.
\end{abstract}

\section{Introduction}

Alkali slag is a by-product of alkali production. The problem of large emissions and difficult stacking has plagued the alkali-making enterprises. In the process of making alkali, about 10 cubic meters of waste liquid is produced per ton of soda ash, and the alkali slag is about 0.3 to 0.6 tons. In 2016, China's soda ash production is 25.83 million tons, an increase of $2.6 \%$ year-on-year, and its alkali slag output is about 776.49 15.924 million tons[1-3]. Such a huge amount of waste will occupy a large area of land if it is piled up, and will pollute the environment if it is directly discharged into the river. Taking Lianyungang Soda Plant as an example, it uses a direct stacking method, covering an area of about 10.1 $\mathrm{Km}^{2}$, which wastes a lot of land area. Figure 1 shows the status of the alkali slag pool. One of the alkali slag pools has been abandoned and is still in normal production and discharge. The waste liquid from the alkali residue is discharged into the tank and then naturally deposited. Therefore, how to resource the use of alkali residue has attracted the attention of many scholars.

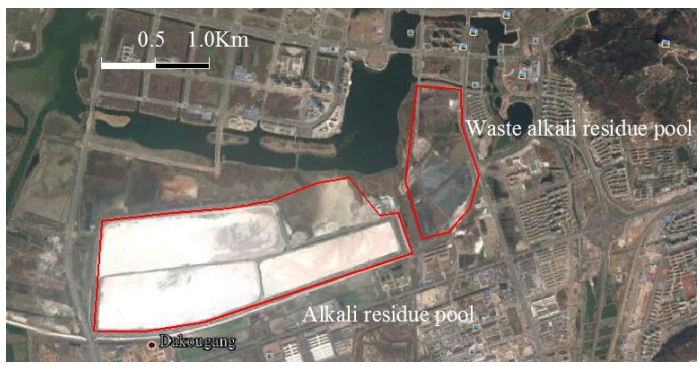

Fig. 1. Aerial view of Lianyungang alkaline slag storage pool

At present, alkali slag is mainly used in the fields of improving acid soil, making desulfurizers, and making fertilizers. Due to the large amount of use in the construction field, more attention has been paid. Current applications include the use of alkali slag to make mortar and block, the production of reinforcing grouting fluid, soil solidifying agent, and alkaline dregs mixed packing.

In this paper, the alkali slag is mainly used to solidify marine soft soil, and relevant experimental research is carried out. The results show that the marine soft soil improved by alkali slag can achieve the engineering properties of general clay, and the alkali residue can be applied to engineering construction.

\section{Properties of alkali residue}

The main components of the alkali residue are $\mathrm{CaCO}_{3}$, $\mathrm{MgCO}_{3}, \mathrm{CaCl}_{2}, \mathrm{NaCl}, \mathrm{Na}_{2} \mathrm{SO}_{4}, \mathrm{CaSO}_{4}$, etc., wherein the content of $\mathrm{CaCO} 3$ and $\mathrm{MgCO} 3$ accounts for more than $60 \%$. From the perspective of particle composition, alkali residue is a poorly graded powder material, and its particle size is mainly distributed in the range of 0.01 to $0.075 \mathrm{~mm}$. The liquid limit of the alkali residue is about $95 \%$ in the natural state, and the plastic limit is about $45 \%$. It can be seen that the alkali residue is a hydrophilic substance. At a pressure of $100 \mathrm{kPa}$ to 200 $\mathrm{kPa}$, the compression modulus of the alkali slag is about $5 \mathrm{MPa}$, and the compressibility is large[4-6].

The SEM analysis shows that the voids in the alkali slag are extremely developed. The forms of voids are intergranular pores, pores between aggregates and pores between aggregates. The connections between mineral particles are loose. This is the root cause of large pores and high water content.

Under the action of high temperature, the properties of the alkali slag will change. As the temperature increases, the looseness of the alkali slag agglomerates decreases, and the density is improved. As the

\footnotetext{
* Corresponding author: Shao Yong, Ph.D., Associate Professor. Mainly engaged in teaching and research work in geotechnical engineering. Email:silinfe@sina.com.
} 
temperature increases, the pores of the alkali slag also increase, but the number of pores decreases. At temperatures up to $600^{\circ} \mathrm{C}$, the surface of the alkali slag particles will undergo significant melting and the bonds between the particles will be tighter[7-8].

In general, alkali slag has the characteristics of large pores, high water content, high liquid-plastic limit, high hydrophilicity, high compressibility and improved mechanical properties under high temperature conditions.

This paper mainly studies the improvement of marine soft soil by alkali slag. Therefore, the basic properties of alkali slag and marine soft soil are given together, as shown in Table 1.

Table 1. The properties of alkali residue and marine soft soil

\begin{tabular}{|c|c|c|}
\hline Index & Alkali residue & Marine soft soil \\
\hline$\rho_{\max } /\left(\mathrm{g} / \mathrm{cm}^{3}\right)$ & 1.01 & 1.58 \\
\hline$w_{\mathrm{op}} / \%$ & 46.5 & 26.1 \\
\hline$w_{\mathrm{\nu}} / \%$ & 90.3 & 60.3 \\
\hline$w_{\mathrm{p}} / \%$ & 37.5 & 30.2 \\
\hline$c_{c u} / k P a$ & 34 & 10 \\
\hline$\phi_{c u} /\left(^{\circ}\right)$ & 15 & 5 \\
\hline
\end{tabular}

\section{Test arrangement}

\subsection{Test plan}

The marine soft soil was taken back to the laboratory for air drying (air dried moisture content of 3.73\%) and pulverized through a $0.5 \mathrm{~mm}$ sieve. As shown in Fig. 2, it is a marine soft soil after sieving. The preparation process of the alkali residue is similar to that of the sludge, and is naturally dried and pulverized, and then passed through a $0.25 \mathrm{~mm}$ sieve, as shown in Fig. 3, as an alkali slag after sieving. The preliminary test is to carry out the curing test by adding alkali slag. The alkali slag content is $6 \%, 12 \%, 18 \%, 24 \%, 30 \%, 36 \%$, and the percentage of the solidified material is the mass percentage of the curing agent and the sludge dry soil.

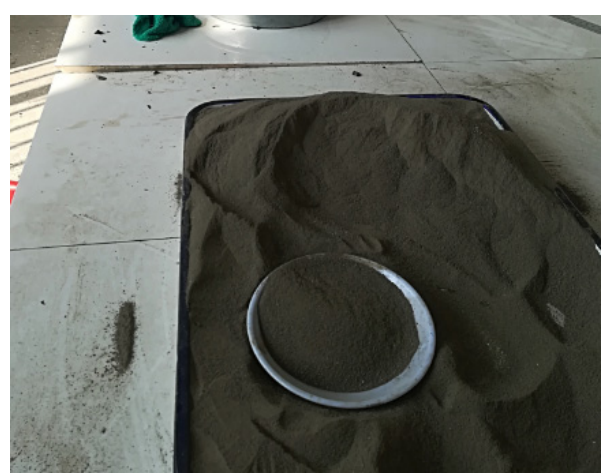

Fig. 2. Mud soil after sieving

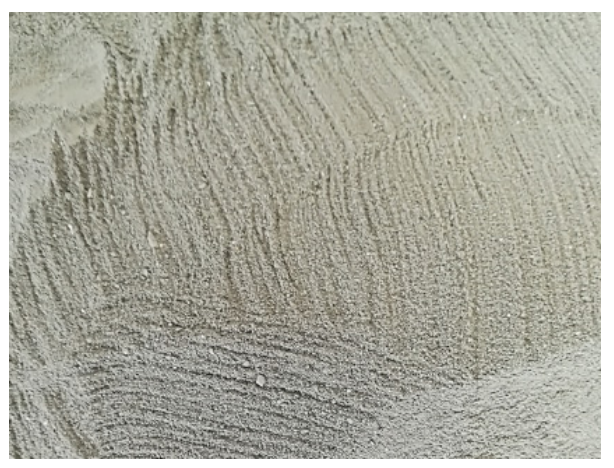

Fig. 3. Alkali residue after sieving

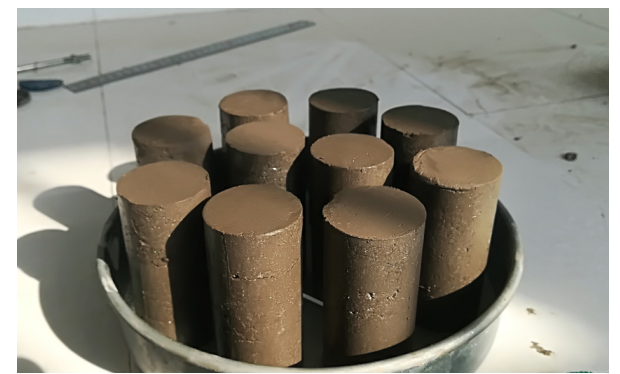

Fig. 4. Alkali slag and marine soft soil mixture sample

\subsection{Basic properties of the mixture}

Using alkaline slag to improve marine soft soil, this paper first tests the basic properties of alkaline slag marine soft soil mixture. That is, the maximum dry density and the optimum moisture content of the mixture. The test results are shown in Table 2 and Figure 3.

Table 2. The properties of alkali residue and marine soft soil

\begin{tabular}{|c|c|c|}
\hline Alkali slag content(\%) & $\rho_{\text {max }} /\left(\mathbf{g} / \mathbf{c m}^{\mathbf{3}}\right)$ & $\boldsymbol{w}_{\text {op }} / \%$ \\
\hline 0 & 1.578 & 26.1 \\
\hline 6 & 1.513 & 27.4 \\
\hline 12 & 1.454 & 28.6 \\
\hline 18 & 1.421 & 30.3 \\
\hline 24 & 1.413 & 31.2 \\
\hline 30 & 1.403 & 32.3 \\
\hline 36 & 1.401 & 33.6 \\
\hline
\end{tabular}

It can be seen that as the alkali slag content increases, the maximum dry density of the mixture decreases, and the rate of decrease decreases only when the alkali slag content is high. This is due to the small density of the alkali slag. 


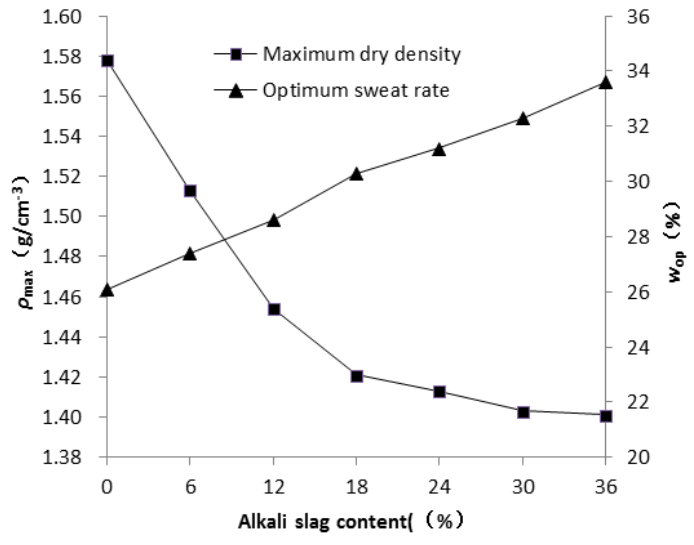

Fig. 5. Alkali slag and marine soft soil mixture sample

\section{Analysis of test results}

\subsection{Destructive feature}

Figure 6 is a picture of the sample loading process. In the middle stage of loading, it can be seen that there are longitudinal cracks on the surface of the sample, and as the loading continues, these longitudinal cracks begin to pass through. Subsequently, the sample is destroyed. After the sample is destroyed, there is generally a penetrating crack, and the crack has a certain inclination angle, which is an oblique crack. During the loading process, it is found that the alkali slag modified soft soil has a certain viscosity, and its failure strain is generally large, generally more than 10\%, as shown in Figure 7.
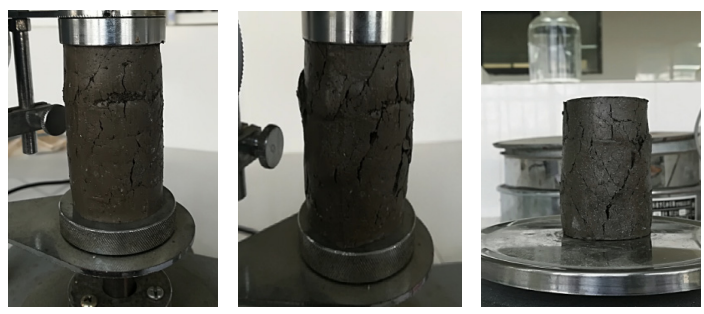

Fig. 6. Sample destruction process

It can be seen from Fig. 7, as the alkali slag content increases, the strain at break decreases. And satisfy the power function relationship.

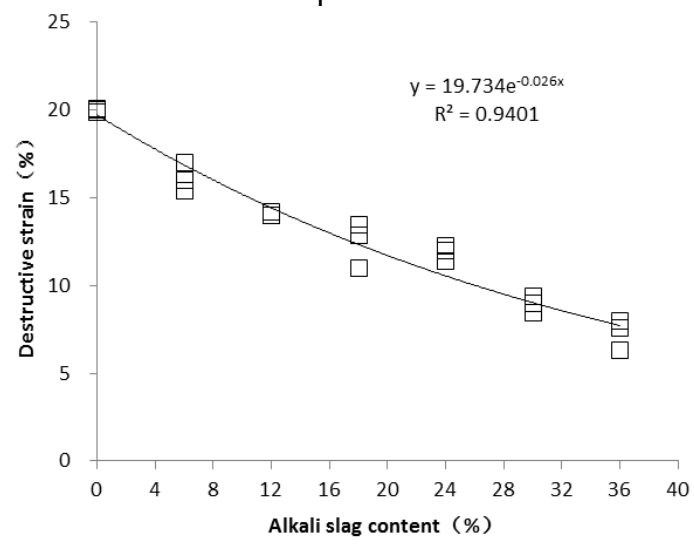

Fig. 7. Relationship between alkali slag content and failure strain

\subsection{Strength test result}

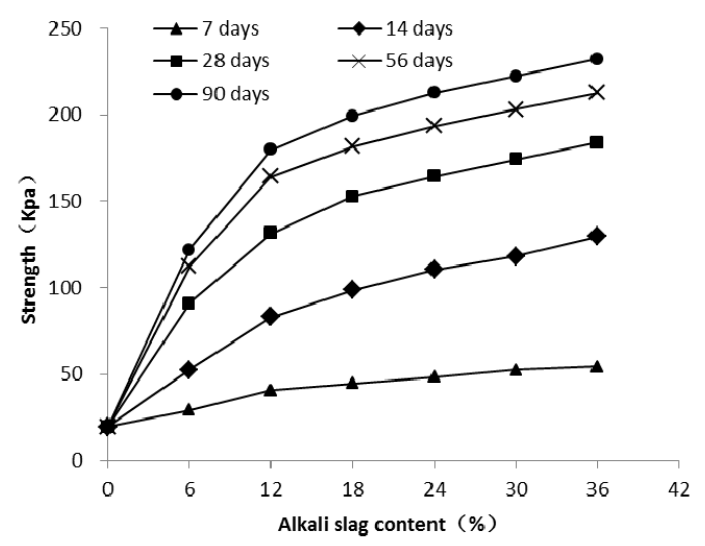

Fig. 8. Unconfined compressive strength test results

After the samples reached a certain age, the unconfined compressive strength test was carried out and tested at 7 days, 14 days, 28 days, 56 days and 90 days. The test results are shown in Figure 8 . It can be seen that as the content of alkali slag increases, the strength of the sample also increases, but by the shape of the curve, the alkali slag content is $12 \%$ as a demarcation point. The increase of the previous strength is larger, and then smaller, indicating that the alkali slag content is a nonlinear relationship to the improvement of the strength of marine soft soil.

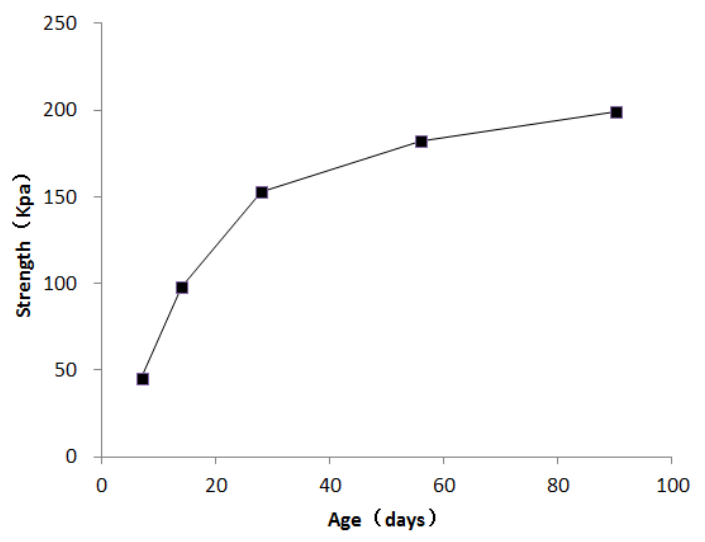

Fig. 9. Strength versus age

Figure 9 shows a sample with an alkali slag content of $18 \%$, giving the relationship between age and strength. It can be seen that after 28 days of age, the increase in the strength of the sample becomes smaller.

\subsection{Compression modulus}

Figure 10 shows the relationship between stress and strain when the sample is loaded. The viscosity characteristics of the sample can be seen, and the less the alkali slag content, the more obvious. 


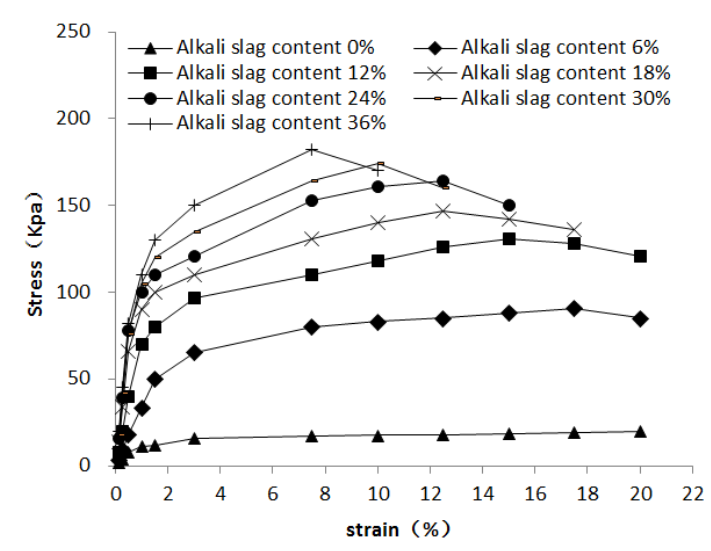

Fig. 10. Stress-strain relationship curve

Figure 11 shows the relationship between the compressive modulus of the sample and the alkali slag content. It can be seen that the compressive modulus of the sample is greatly improved after the alkali slag is incorporated into the marine soft soil. The maximum value in the figure is $18.12 \mathrm{MPa}$. The level of general clay has been reached. In addition, similar to the strength, the alkali slag content of $18 \%$ is still a cut-off point, and the previous compression modulus is increased faster and then becomes slower. It is also a nonlinear relationship.

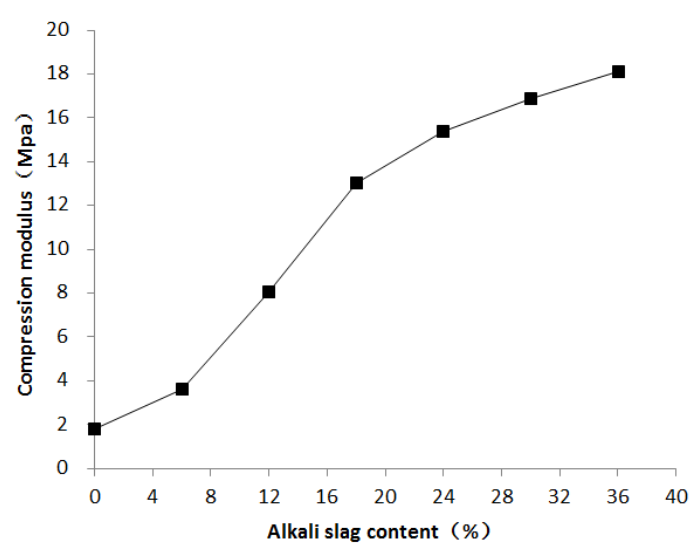

Fig. 11. Relationship between compressive modulus and alkali slag content

\subsection{Softening coefficient}

In order to analyze the engineering characteristics of the improved soil after water, the softening coefficient test was also carried out. Samples of 14 days, 28 days, and 56 days age were selected for testing. Two samples were taken out for two days before the specified age, and two samples were taken out from the curing box two days later. The compressive strength was measured together with two samples immersed in water for 48 hours, and the softening coefficient of the modified soil was calculated. The result is shown in Figure 12.

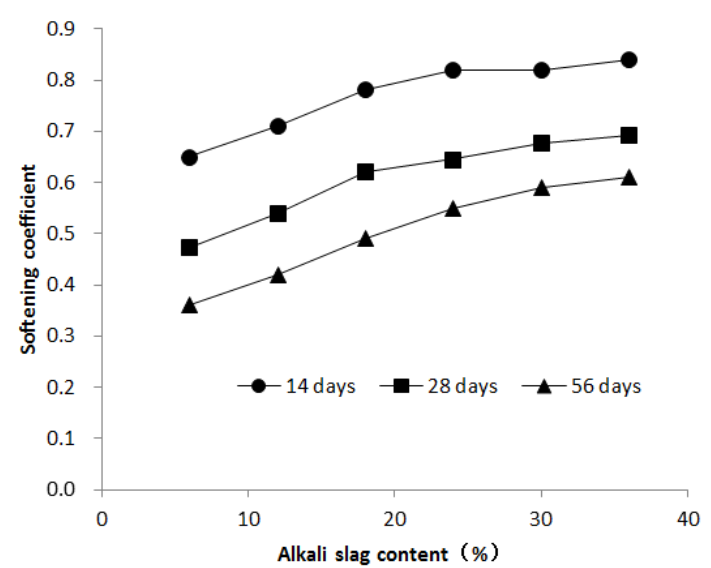

Fig. 12. Relationship between softening coefficient and alkali residue content

It can be seen that the smaller the age, the greater the softening coefficient will be, and the greater the age, the smaller the softening coefficient will be. The minimum value of the 56-day age is 0.36 . Alkali slag content also has an effect on the softening coefficient. It can be seen from the figure that the larger the alkali slag content, the greater the softening coefficient will be, indicating that the alkali slag can improve the water-receiving characteristics of the marine soft soil and improve the strength stability. In the soft soil coefficient test, there is also a critical value of $18 \%$, which is consistent with the above test results.

\section{Conclusions}

The paper uses alkaline slag to improve the marine soft soil. Through experimental analysis, the following conclusions are obtained.

(1) Alkali slag is an industrial by-product and is also a burden to the environment. It is used to improve the marine soft soil and it is proved to be feasible through experimental analysis.

(2) Alkali slag modified soil has a certain viscosity, which is reflected in the damage characteristics. The strained sample is concentrated in the paper, and its failure strain is about $10 \%$.

(3) When the alkaline slag is added to the marine soft soil, the unconfined compression resistance and the compression modulus are both increased by about 10 times. It shows that the improvement effect is remarkable. This can be applied in engineering practice.

\section{References}

1. FANG YING-guang, ZHU Zhong-wei, Mo Haihong, et al. Experimental Research on Vibration and Drainage Consolidation of Caustic. CHIN JRM, 01, 43(2008).

2. CAO Jun, JIANG Hai-bin, ZHANG Yuan. Physical and Chemical Properties of Alkali Plant Caustic Soda and Engineering Properties. SICI, 45, 37(2016). 
3. WANG Fang, XU Zhu-qing, YAN Li-xue, et al. Experimental Test Method of Alkaline Dross and Its Engineering Soil Characteristics. CHIN JRM, 08, 1211(2007).

4. YAN Chi, LI Lin, SUN Hong-yue. Compressive Deformation of Alkali Residue. J QACE, 04, 08(2003) .

5. ZHAO Hong-liang. Experimental Research on Engineering Properties of Industrial Waste Alkaliearth. GT, 04, 49(2000).

6. Li Lin, JIANG Zhi-an. Experimental Study on the Deformation Properties of Caustic Soda. HGE, 05, 77(2005).

7. YANG Yi-bo, PU Yong-qiang, YAN Wei-jun, et al. Microstructure and Chloride ion Elution Characteristics of Caustic Sludge. J SCUT, 45, 82(2017).

8. SUN Jiaying, GU Xin. Engineering Characteristics of a New Clinker-Free Alkali-Stabilized Soi. CHIN J BM, 17, 1031(2014). 